\title{
VARIATIONAL ITERATION METHOD FOR DELAY DIFFERENTIAL-ALGEBRAIC EQUATIONS
}

\author{
Hongliang Liu ${ }^{1}$, Aiguo Xiao ${ }^{1}$, Yongxiang Zhao ${ }^{1,2}$ \\ ${ }^{1}$ School of Mathematics and Computational Science, Xiangtan University, \\ Hunan 411105, China, lhl@xtu.edu.cn,xag@xtu.edu.cn \\ ${ }^{2}$ School of Mathematics and Computer Science, Three Gorges University, \\ Chongqing 404000, China, zyxlily80@126.com
}

\begin{abstract}
Variational iteration method is applied to solve a class of delay differentialalgebraic equations. The obtained sequence of iteration is based on the use of Lagrange multipliers. The corresponding convergence results are obtained and successfully confirmed by some numerical examples.
\end{abstract}

Keywords- Delay Differential-Algebraic Equations, Variational Iteration Method, Convergence

\section{INTRODUCTION}

The variational iteration method (VIM) was first proposed by He [1, 2], and has been extensively discussed by many authors [3-10]. Applications of this method have been enlarged due to its flexibility, convenience and efficiency. Some authors have applied VIM to delay differential equations [7] and differential-algebraic equations [3], but VIM for delay differential-algebraic equations (DDAEs) has not been considered. In fact, DDAEs are a very important class of mathematical models and often arise from the fields of computer aided design, circuit analysis, mechanical systems, etc. Some results in theoretical analysis and numerical solutions of DDAEs have been given, which include stability of Runge-Kutta methods for neutral delay integro-differential-algebraic equations [11], the classical convergence results of BDF methods and Runge-Kutta methods for index-2 DDAEs [12] and collocation methods for retarded differentialalgebraic equations [13]. In this paper, we apply VIM to a class of DDAEs to obtain approximate analytical solutions. The convergence results of the VIM for DDAEs are obtained. Some illustrative examples confirm the theoretical results.

\section{MAIN RESULTS}

Consider the initial value problem of a DDAE

$$
\left\{\begin{array}{lr}
x^{\prime}(t)=f(x(t), x(\alpha(t)), y(t), y(\beta(t))), & 0 \leq t \leq T, \\
0=g(x(t), x(\alpha(t)), y(t)), & 0 \leq t \leq T, \\
x(t)=\varphi(t),-\tau_{1} \leq t \leq 0, \quad y(t)=\psi(t), & -\tau_{2} \leq t \leq 0,
\end{array}\right.
$$

where the delay functions $\alpha(t)$ and $\beta(t)$ satisfy $-\tau_{1} \leq \alpha(t) \leq t, \quad-\tau_{2} \leq \beta(t) \leq t, \quad f: R^{n_{1}}$ 
$\times R^{n_{1}} \times R^{n_{2}} \times R^{n_{2}} \rightarrow R^{n_{1}}, g: R^{n_{1}} \times R^{n_{1}} \times R^{n_{2}} \rightarrow R^{n_{2}}$ are smooth vector functions on the real Euclidean spaces and have bounded derivatives, the initial value functions $\varphi:\left[-\tau_{1}, 0\right]$ $\rightarrow R^{n_{1}}$ and $\psi:\left[-\tau_{2}, 0\right] \rightarrow R^{n_{2}}$ are continuous, $g_{y}(x(t), x(\alpha(t)), y(t))$ is invertible and bounded in a neighbourhood of the true solution. We assume that the problem (1) has a smooth solution $x(t), y(t)$. Throughout this paper, $\|\cdot\|$ denotes the standard Euclidean norm, and the matrix norm is subordinate to $\|\cdot\|$.

According to the VIM, we can construct the correction functional as follows

$$
\begin{aligned}
& x_{n+1}(t)=x_{n}(t)+\int_{0}^{t} \lambda(s, t)\left(x_{n}^{\prime}(s)-\widetilde{f}\left(x_{n}(s), x_{n}(\alpha(s)), y_{n}(s), y_{n}(\beta(s))\right)\right) d s, \\
& 0=g\left(x_{n+1}(t), x_{n+1}(\alpha(t)), y_{n+1}(t)\right),
\end{aligned}
$$

where $\lambda(s, t)$ is a general Lagrange multiplier, which can be defined optimally by variational theory, and $\widetilde{f}$ denotes the restrictive variation, i.e., $\delta \widetilde{f}=0$. Thus, we have

$$
\delta x_{n+1}(t)=\delta x_{n}(t)+\int_{0}^{t} \lambda(s, t)\left(\delta x_{n}^{\prime}(s)\right) d s,
$$

and the stationary conditions are obtained as

$$
1+\left.\lambda(s, t)\right|_{s=t}=0, \quad \frac{\partial \lambda(s, t)}{\partial s}=0 .
$$

Moreover, the general Lagrange multiplier can be readily identified by $\lambda(s, t)=-1$. Therefore, the variational iteration formula can be written as

$$
\begin{aligned}
& x_{n+1}(t)=x_{n}(0)+\int_{0}^{t} f\left(x_{n}(s), x_{n}(\alpha(s)), y_{n}(s), y_{n}(\beta(s))\right) d s, \\
& 0=g\left(x_{n+1}(t), x_{n+1}(\alpha(t)), y_{n+1}(t)\right) .
\end{aligned}
$$

Theorem 1 Let $x(t), x_{i}(t) \in\left(C^{1}\left[-\tau_{1}, T\right]\right)^{n_{1}}, y(t), y_{i}(t) \in\left(C^{1}\left[-\tau_{2}, T\right]\right)^{n_{2}}, i=0,1, \cdots$. Then the sequences $\left\{x_{n}(t)\right\}_{n=1}^{\infty},\left\{y_{n}(t)\right\}_{n=1}^{\infty}$ defined by (3) with $x_{0}(t)=\varphi(t),-\tau_{1} \leq t \leq 0$, $y_{0}(t)=\psi(t),-\tau_{2} \leq t \leq 0$ converge to the solution of (1).

Proof. From the system (1), we obviously have

$$
\begin{aligned}
& x(t)=x(0)+\int_{0}^{t} f(x(s), x(\alpha(s)), y(s), y(\beta(s))) d s, \\
& 0=g(x(t), x(\alpha(t)), y(t)) .
\end{aligned}
$$

Introduce $E_{i} x(t)=x_{i}(t)-x(t), E_{i} y(t)=y_{i}(t)-y(t), i=0,1, \cdots$, where $E_{i} x(t)=E_{i} y(t)$ $=0, t<0, i=0,1, \cdots$. From (3)-(4), we obtain

$$
\begin{aligned}
& E_{n+1} x(t)=\int_{0}^{t}\left(f\left(x_{n}(s), x_{n}(\alpha(s)), y_{n}(s), y_{n}(\beta(s))\right)-f(x(s), x(\alpha(s)), y(s), y(\beta(s)))\right) d s, \\
& 0=g\left(x_{n+1}(t), x_{n+1}(\alpha(t)), y_{n+1}(t)\right)-g(x(t), x(\alpha(t)), y(t)) .
\end{aligned}
$$


Based on the fact that the functions $f, g$ are smooth and the matrix $g_{y}$ is invertible, we deduce

$$
\begin{aligned}
& E_{n+1} x(t)=\int_{0}^{t}\left(f_{1}^{\prime} E_{n} x(s)+f_{2}^{\prime} E_{n} x(\alpha(s))+f_{3}^{\prime} E_{n} y(s)+f_{4}^{\prime} E_{n} y(\beta(s))\right) d s, \\
& E_{n+1} y(t)=-\left(g_{3}^{\prime}\right)^{-1} g_{1}^{\prime} E_{n+1} x(t)-\left(g_{3}^{\prime}\right)^{-1} g_{2}^{\prime} E_{n+1} x(\alpha(t)),
\end{aligned}
$$

where $f_{i}^{\prime}(i=1,2,3,4)$ denotes the partial derivative of the function $f$ to ith variable, $g_{i}^{\prime}(i=1,2,3)$ denotes the partial derivative of the function $g$ to ith variable. We can derive

$$
\left(\begin{array}{l}
\left\|E_{n+1} x(t)\right\| \\
\left\|E_{n+1} y(t)\right\|
\end{array}\right) \leq\left(\begin{array}{cc}
l_{1} & l_{3} \\
2 k l_{1} & 2 k l_{3}
\end{array}\right)\left(\begin{array}{l}
\int_{0}^{t}\left\|E_{n} x(s)\right\| d s \\
\int_{0}^{t}\left\|E_{n} y(s)\right\| d s
\end{array}\right)+\left(\begin{array}{cc}
l_{2} & l_{4} \\
2 k l_{2} & 2 k l_{4}
\end{array}\right)\left(\begin{array}{l}
\int_{0}^{t}\left\|E_{n} x(\alpha(s))\right\| d s \\
\int_{0}^{t}\left\|E_{n} y(\beta(s))\right\| d s
\end{array}\right),
$$

where $l_{i}=\max \left\|f_{i}^{\prime}\right\|,(i=1,2,3,4), \quad k=\max \left(\left\|\left(g_{3}^{\prime}\right)^{-1} g_{1}^{\prime}\right\|,\left\|\left(g_{3}^{\prime}\right)^{-1} g_{2}^{\prime}\right\|\right)$. Therefore

$$
\begin{aligned}
\left(\begin{array}{l}
\left\|E_{1} x(t)\right\| \\
\left\|E_{1} y(t)\right\|
\end{array}\right) & \leq\left(\begin{array}{cc}
l_{1} & l_{3} \\
2 k l_{1} & 2 k l_{3}
\end{array}\right)\left(\begin{array}{l}
\int_{0}^{t}\left\|E_{0} x(s)\right\| d s \\
\int_{0}^{t}\left\|E_{0} y(s)\right\| d s
\end{array}\right)+\left(\begin{array}{cc}
l_{2} & l_{4} \\
2 k l_{2} & 2 k l_{4}
\end{array}\right)\left(\begin{array}{l}
\int_{0}^{t}\left\|E_{0} x(\alpha(s))\right\| d s \\
\int_{0}^{t}\left\|E_{0} y(\beta(s))\right\| d s
\end{array}\right), \\
& \leq\left(\begin{array}{cc}
l_{1}+l_{2} & l_{3}+l_{4} \\
2 k\left(l_{1}+l_{2}\right) & 2 k\left(l_{3}+l_{4}\right)
\end{array}\right)\left(\begin{array}{c}
\max _{-\tau_{1} \leq s \leq T}\left\|E_{0} x(s)\right\| t \\
\max _{-\tau_{2} \leq s \leq T}\left\|E_{0} y(s)\right\| t
\end{array}\right) .
\end{aligned}
$$

Moreover, we have

$$
\left(\begin{array}{l}
\left\|E_{n} x(t)\right\| \\
\left\|E_{n} y(t)\right\|
\end{array}\right) \leq \frac{(\tau+T)^{n} \rho^{n}}{n !}\left(\begin{array}{c}
\max _{-\tau_{1} \leq s \leq T}\left\|E_{0} x(s)\right\| \\
\max _{-\tau_{2} \leq s \leq T}\left\|E_{0} y(s)\right\|
\end{array}\right) .
$$

where $\tau, T, \max _{-\tau_{1} \leq s \leq T}\left\|E_{0} x(s)\right\|, \max _{-\tau_{2} \leq s \leq T}\left\|E_{0} y(s)\right\|, k, l_{i}$, $(i=1,2,3,4)$ are constants, $\rho$ is the spectral radius of the last matrix in the above inequality (6). By using the Stirling's formula, we have

$$
\left(\begin{array}{l}
\left\|E_{n} x(t)\right\| \\
\left\|E_{n} y(t)\right\|
\end{array}\right) \leq \frac{\left(\frac{(\tau+T) \rho e}{n}\right)^{n}}{\sqrt{2 n \pi}(1+O(1 / n))}\left(\begin{array}{c}
\max _{-\tau_{1} \leq s \leq T}\left\|E_{0} x(s)\right\| \\
\max _{-\tau_{2} \leq s \leq T}\left\|E_{0} y(s)\right\|
\end{array}\right),
$$

thus, $\left(\left\|E_{n} x(t)\right\|,\left\|E_{n} y(t)\right\|\right)^{T} \rightarrow 0$ as $n \rightarrow \infty$. 


\section{ILLUSTRATIVE EXAMPLES}

In this section, some illustrative examples are given to show the efficiency of the VIM for DDAEs.

Example 1 Consider the following initial value problem

$$
\begin{cases}y^{\prime}(t)=y^{2}(t)-2 y^{2}\left(\frac{t}{2}\right)+x^{2}\left(\frac{t}{2}\right), & t \geq 0 \\ 1=2 y^{2}\left(\frac{t}{2}\right)+x(t), & t \geq 0 \\ x(0)=1, y(0)=0 . & \end{cases}
$$

We apply the VIM to (9), and construct the correction functional

$$
\begin{aligned}
& y_{n+1}(t)=y_{n}(0)+\int_{0}^{t}\left(y_{n}^{2}(s)-2 y_{n}^{2}\left(\frac{s}{2}\right)+x_{n}^{2}\left(\frac{s}{2}\right)\right) d s, \\
& 1=2 y_{n+1}^{2}\left(\frac{t}{2}\right)+x_{n+1}(t) .
\end{aligned}
$$

Moreover, the iteration sequence starts with the initial approximations $y_{0}(t)=t$, $x_{0}(t)=x(0)=1$, and is obtained from (10) as follows

$$
\begin{aligned}
& y_{1}(t)=t+\frac{1}{6} t^{3} \\
& x_{1}(t)=1-2 t^{2}-\frac{2}{3} t^{4}-\frac{1}{18} t^{6}, \\
& y_{2}(t)=t-\frac{1}{6} t^{3}+\frac{11}{120} t^{5}+\frac{11}{1152} t^{7}+o\left(t^{7}\right), \\
& x_{2}(t)=1-2 t^{2}+\frac{2}{3} t^{4}-\frac{19}{45} t^{6}+\frac{11}{480} t^{8}+o\left(t^{8}\right), \\
& y_{3}(t)=t-\frac{1}{6} t^{3}+\frac{1}{120} t^{5}+\frac{431}{20160} t^{7}-\frac{13}{40960} t^{9}+o\left(t^{9}\right), \\
& x_{3}(t)=1-2 t^{2}+\frac{2}{3} t^{4}-\frac{6}{45} t^{6}-\frac{403}{5040} t^{8}+\frac{148861}{9676800} t^{10}+o\left(t^{10}\right),
\end{aligned}
$$

From the above iteration sequence, we can show that

$$
\lim _{n \rightarrow \infty} y_{n}(t)=\sin (t), \quad \lim _{n \rightarrow \infty} x_{n}(t)=\cos (2 t) .
$$

Example 2 Consider the following initial value problem

$$
\begin{cases}x^{\prime}(t)=-2 y\left(\frac{t}{2}\right)+2 z(t), & t \geq 0 \\ y^{\prime}(t)=-2 x(t) z^{2}\left(\frac{t}{2}\right)+z^{2}(t), & t \geq 0 \\ 0=y(t)-x(t) z(t), & t \geq 0 \\ x(0)=1, \quad y(0)=0, \quad z(0)=1 . & \end{cases}
$$

The exact solution of the system (12) is

$$
\begin{aligned}
& x(t)=(1+t) e^{-t}=1-\frac{1}{2} t^{2}+\frac{1}{3} t^{3}-\frac{1}{8} t^{4}+\frac{1}{30} t^{5}+o\left(t^{5}\right), \\
& y(t)=(1+t) e^{-2 t}=1-t+\frac{2}{3} t^{3}-\frac{2}{3} t^{4}+\frac{2}{5} t^{5}+o\left(t^{5}\right), \\
& z(t)=e^{-t}=1-t+\frac{1}{2} t^{2}-\frac{1}{6} t^{3}+\frac{1}{24} t^{4}-\frac{1}{120} t^{5}+o\left(t^{5}\right) .
\end{aligned}
$$


We apply the VIM to (12), and construct the correction functional

$$
\begin{aligned}
& x_{n+1}(t)=x_{n}(0)+\int_{0}^{t}\left(-2 y_{n}\left(\frac{s}{2}\right)+2 z_{n}(s)\right) d s, \\
& y_{n+1}(t)=y_{n}(0)+\int_{0}^{t}\left(-2 x_{n}(s) z_{n}^{2}\left(\frac{s}{2}\right)+z_{n}^{2}(s)\right) d s, \\
& 0=y_{n+1}(t)-x_{n+1}(t) z_{n+1}(t) .
\end{aligned}
$$

Moreover, the iteration sequence starts with the initial approximations $x_{0}(t)=x(0)=1$, $y_{0}(t)=y(0)=1, z_{0}(t)=z(0)=1$, and is obtained from (13) as follows

$$
\begin{aligned}
& x_{1}(t)=1 \\
& y_{1}(t)=1-t \\
& z_{1}(t)=1-t \\
& x_{2}(t)=1-\frac{1}{2} t^{2} \\
& y_{2}(t)=1-t+\frac{1}{6} t^{3} \\
& z_{2}(t)=1-t+\frac{1}{2} t^{2}-\frac{1}{3} t^{3}+\frac{1}{4} t^{4}+o\left(t^{4}\right) \\
& x_{3}(t)=1-\frac{1}{2} t^{2}+\frac{1}{3} t^{3}+\frac{1}{96} t^{4} \\
& y_{3}(t)=1-t+\frac{2}{3} t^{3}-\frac{7}{16} t^{4}+\frac{23}{160} t^{5}+o\left(t^{5}\right), \\
& z_{3}(t)=1-t+\frac{1}{2} t^{2}-\frac{1}{6} t^{3}+\frac{5}{32} t^{4}-\frac{7}{60} t^{5}+o\left(t^{5}\right), \\
& x_{4}(t)=1-\frac{1}{2} t^{2}+\frac{1}{3} t^{3}-\frac{1}{8} t^{4}+\frac{7}{640} t^{5}-\frac{23}{15360} t^{6}+o\left(t^{6}\right), \\
& y_{4}(t)=1-t+\frac{2}{3} t^{3}-\frac{2}{3} t^{4}+\frac{163}{480} t^{5}+\frac{65}{576} t^{6}+o\left(t^{6}\right), \\
& z_{4}(t)=1-t+\frac{1}{2} t^{2}-\frac{1}{6} t^{3}+\frac{1}{24} t^{4}-\frac{89}{1920} t^{5}+\frac{197}{5120} t^{6}+o\left(t^{6}\right),
\end{aligned}
$$

The above iterate sequence shows that the VIM yields a very good approximation to the exact solution.

\section{CONCLUSIONS}

In this paper, we successfully apply VIM to a class of DDAEs and obtain highly accurate solutions with few iterations. VIM handles DDAEs without any especial assumption on the delay item, thus, it is a promising method for DDAEs.

\section{ACKNOWLEDGEMENT}

This work is supported by projects from NSF of China (No.10971175), Specialized Research Fund for the Doctoral Program of Higher Education of China (No.20094301110001), NSF of Hunan Province (No.09JJ3002) and Research Projects of Xiangtan University (No.08xz05). 


\section{REFERENCES}

1. J. H. He, Variational iteration method - a kind of non-linear analytical technique: Some examples, International Journal of Nonlinear Mechanics, 34, 699-708, 1999.

2. J. H. He, Approximate solution of nonlinear differential equations with convolution product nonlinearities, Computer Methods in Applied Mechanics and Engineering 167, 69-73, 1998.

3. F. Soltanian, S. M. Karbassi, M. M. Hosseini, Application of He's variational iteration method for solution of differential-algebraic equations, Chaos, Solitons and Fractals 41, 436-445, 2009.

4. D. K. Salkuyeh, Convergence of the variational iteration method for solving linear systems of ODEs with constant coefficients, Computers and Mathematics with Applications 56, 2027-2033, 2008.

5. A. M. Wazwaz, The variational iteration method for analytic treatment for linear and nonlinear ODEs, Applied Mathematics and Computation 212, 120-134, 2009.

6. A. Saadatmandi, M. Dehghan, Variational iteration method for solving a generalized pantograph equation, Computers and Mathematics with Applications 58, 2190-2196, 2009.

7. Z. H. Yu, Variational iteration method for solving the multi-pantograph delay equation, Physics Letters A 372, 6475-6479, 2008.

8. R. Mokhtari, M. Mohammadi, Some Remarks on the Variational Iteration Method, International Journal of Nonlinear Sciences and Numerical Simulation, 10, 67-74, 2009

9. S. M. Goh, M. S. N. Noorani, I. Hashim, et al. Variational Iteration Method as a Reliable Treatment for the Hyperchaotic Rossler System , International Journal of Nonlinear Sciences and Numerical Simulation 10, 363-371,2009

10. I. Ates , A. Yildirim, Application of variational iteration method to fractional initialvalue problems , International Journal of Nonlinear Sciences and Numerical Simulation 10, 877-883, 2009

11. Y. Xu, J. J. Zhao, Stability of Runge-Kutta methods for neutral delay integro differential-algebraic system, Mathematics and Computers in Simulation 79, 571-583, 2008.

12. U. M. Ascher, L. R. Pezold, The numerical solution of delay-differential-algebraic equations of retarded and neutral type, SIAM Journal on Numerical Analysis 32, 1635-1657, 1995.

13. R. Hauber, Numerical treatment of retarded differential-algebraic equations by collocation methods, Advances in Computational Mathematics 7, 573-592, 1997. 